\title{
Fecal Calprotectin: A Reliable Predictor of Mucosal Healing after Treatment for Active Ulcerative Colitis
}

\author{
Vendel Kristensen, ${ }^{1,2}$ Arne Røseth, ${ }^{3}$ Tahir Ahmad, ${ }^{3}$ Viggo Skar, ${ }^{1}$ and Bjørn Moum ${ }^{2,4}$ \\ ${ }^{1}$ Unger-Vetlesen's Institute, Lovisenberg Diaconal Hospital, Oslo, Norway \\ ${ }^{2}$ Department of Gastroenterology, Oslo University Hospital, Oslo, Norway \\ ${ }^{3}$ Department of Medicine, Lovisenberg Diaconal Hospital, Oslo, Norway \\ ${ }^{4}$ Institute of Clinical Medicine, University of Oslo, Oslo, Norway
}

Correspondence should be addressed to Vendel Kristensen; vendel@vikenfiber.no

Received 21 June 2017; Revised 14 August 2017; Accepted 6 September 2017; Published 31 October 2017

Academic Editor: Per Hellström

Copyright ( 2017 Vendel Kristensen et al. This is an open access article distributed under the Creative Commons Attribution License, which permits unrestricted use, distribution, and reproduction in any medium, provided the original work is properly cited.

\begin{abstract}
Objectives. Mucosal healing has become the new goal of treatment in ulcerative colitis. Fecal calprotectin has been demonstrated to differentiate between mucosal inflammation and mucosal healing. With this project, we investigated whether a reduction in $\mathrm{f}$-calprotectin to $<250 \mu \mathrm{g} / \mathrm{g}$ after medical treatment for active ulcerative colitis could predict mucosal healing. Material and Methods. After a baseline colonoscopy, 20 patients with active ulcerative colitis were followed with consecutive fecal calprotectin monthly until two measurements of fecal calprotectin $<250 \mu \mathrm{g} / \mathrm{g}$ or a maximum follow-up of 12 months. A flexible sigmoidoscopy was then performed and Mayo endoscopic subscore was used to evaluate degree of inflammation. Simple Clinical Colitis Activity Index was used for evaluation of clinical disease activity. Results. A total of 16 patients achieved fecal calprotectin $<250 \mu \mathrm{g} / \mathrm{g}$ during follow-up, and all 16 patients had endoscopic mucosal healing (Mayo endoscopic subscore of $\leq 1$ ) on the second endoscopy. The remaining four patients had persistently high f-calprotectin levels before the second endoscopy with Mayo endoscopic subscore corresponding to endoscopic mucosal healing in three out of four patients. Conclusions. Fecal calprotectin $<250 \mu \mathrm{g} / \mathrm{g}$ after medical treatment for active ulcerative colitis is a reliable marker of endoscopic mucosal healing.
\end{abstract}

\section{Introduction}

Ulcerative colitis (UC) is a chronic disease characterized by intestinal inflammation. The clinical course is typical with periods of remission interrupted by episodes of relapse. Traditionally, treatment of UC aimed to reduce the inflammation during relapse and maintain control of symptoms. Endoscopic remission reduces the risk of clinical relapse, hospitalization, and future colectomy in UC [1-3], leading to mucosal healing as the new goal of treatment in recent years. Fecal- (f-) calprotectin is demonstrated to correlate well with endoscopic disease activity, in contrast to clinical activity indices and inflammatory markers such as Creactive protein, erythrocyte sedimentation rate, and leukocytes [4-6]. Consecutive f-calprotectin measurements to evaluate response to medical treatment in clinical trials and during clinical follow-up represent an interesting noninvasive strategy, but require further studies.

A recent study evaluated the effect of infliximab induction therapy in patients with UC using weekly f-calprotectin measurements for ten weeks and found f-calprotectin less than $50 \mu \mathrm{g} / \mathrm{g}$ to correlate excellently with endoscopic remission defined as Mayo endoscopic subscore $\leq 1$ [7]. The optimal f-calprotectin cut-off level separating endoscopic active UC from UC in endoscopic remission is though probably higher than $50 \mu \mathrm{g} / \mathrm{g}$ and depends on the assay used for analysis $[8,9]$.

Our aim in this study was to evaluate if f-calprotectin below a predefined cut-off level after treatment for active UC was a reliable biomarker of endoscopic mucosal healing. 


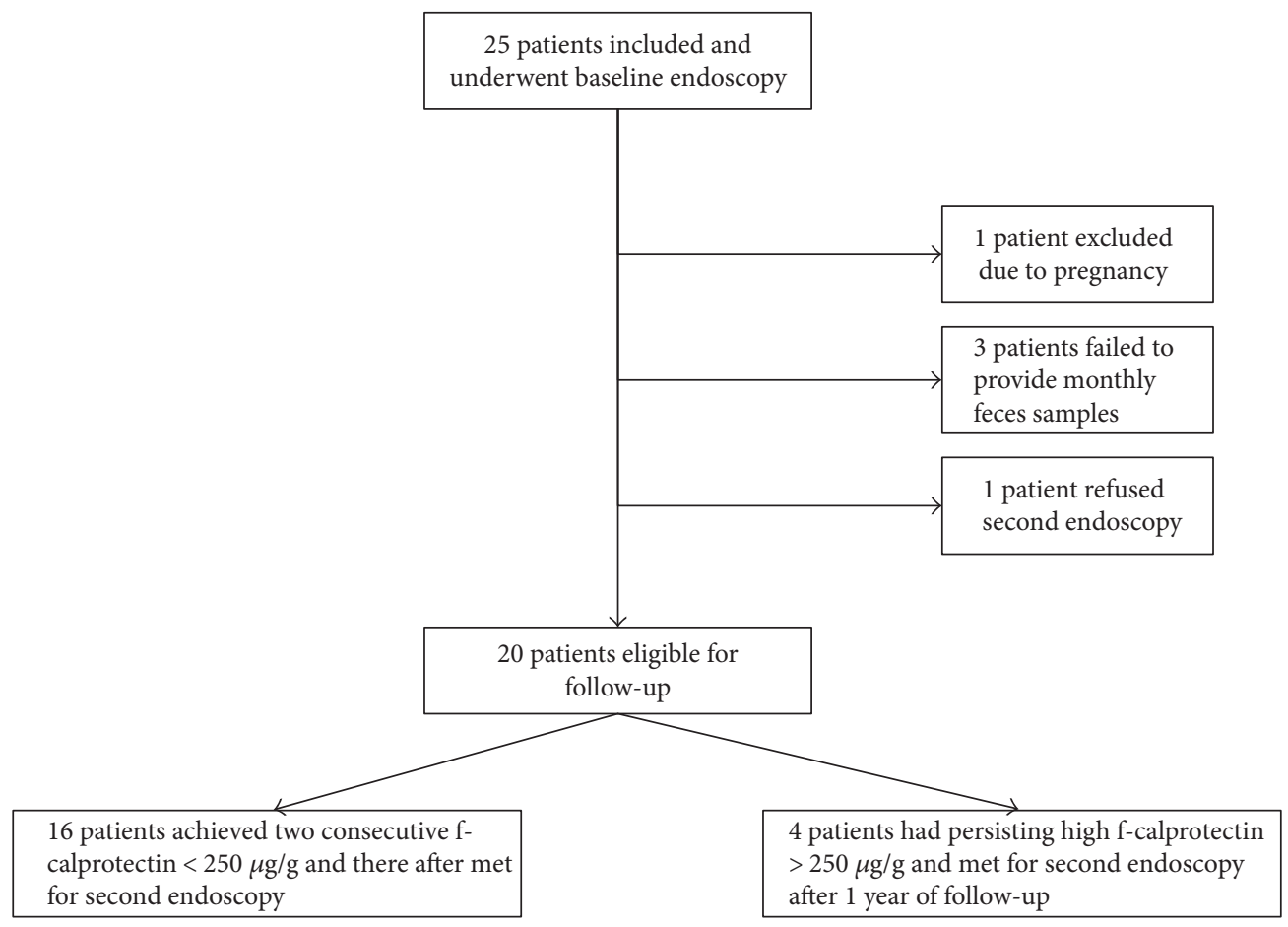

FIGURE 1: Flow chart of patients included in the study.

\section{Materials and Methods}

2.1. Patients. Between December 2012 and October 2014, adult patients with active UC were included in the study. Both newly diagnosed patients and patients with a flare of established disease were invited to participate.

2.2. $f$-calprotectin. The patients were instructed to provide a feces sample 3 days after the baseline colonoscopy and then monthly during follow-up.

The feces samples were stored at $-20^{\circ} \mathrm{C}$ until analysis by an enzyme-linked immunosorbent assay (ELISA) (Bühlmann Laboratories AG, Schönenbuch, Switzerland) according to the manufacturer's instructions. The analytical range was between 30 and $1800 \mu \mathrm{g} / \mathrm{g}$. Samples with $<30 \mu \mathrm{g} / \mathrm{g}$ were registered as $29 \mu \mathrm{g} / \mathrm{g}$ and $>1800 \mu \mathrm{g} / \mathrm{g}$ as $1801 \mu \mathrm{g} / \mathrm{g}$.

2.3. Assessment of Disease Activity. All patients had a colonoscopy performed at inclusion. Montreal classification for disease extent [10] was registered and endoscopic disease activity assessed using the Mayo endoscopic subscore [11]. The segment with the most severe inflammatory activity was chosen to set the score. After two consecutive fcalprotectin measurements $<250 \mu \mathrm{g} / \mathrm{g}$, or after one year of follow-up without achieving two consecutive f-calprotectin measurements $<250 \mu \mathrm{g} / \mathrm{g}$, a flexible sigmoidoscopy was performed. A flexible sigmoidoscopy was chosen as the second endoscopy, as this is less invasive than a full colonoscopy. Three days after the second endoscopy, the patients were asked to send another feces sample.
Routine serological markers and Simple Clinical Colitis Activity Index (SCCAI) [12] were registered at the time of the first and second endoscopy.

2.4. Statistical Analyses. Data are presented as medians with interquartile ranges (IQR) unless otherwise specified. Clinical performance characteristics are presented as point estimates of the mean with $95 \%$ confidence intervals (CI).

Statistical analyses were performed using IBM SPSS Statistics version 22 (SPSS Inc., Chicago, IL).

2.5. Ethical Considerations. The study was approved by the Norwegian South East Regional Committee for Medical and Health Research Ethics. All study participants signed an informed consent before entering the study.

\section{Results}

Altogether, 25 UC patients were included. During the followup, five patients were excluded or lost to follow-up (Figure 1). Baseline characteristics for the 20 patients included in the analysis are presented in Table 1.

f-calprotectin values for all patients during the study period are presented in Figure 2. During follow-up, 16 patients achieved two consecutive f-calprotectin measurements $<250 \mu \mathrm{g} / \mathrm{g}$. The duration from baseline endoscopy and until f-calprotectin $<250 \mu \mathrm{g} / \mathrm{g}$ was reached, ranging from two to 10 months, with a median of four months. All 16 patients had at the follow-up sigmoidoscopy a Mayo endoscopic subscore of $\leq 1$. Two out of these 16 patients had a SCCAI score of $>2.5$ at the time of the second endoscopy. 
TABLE 1: Baseline characteristics of the study participants.

\begin{tabular}{lc}
\hline Number of patients & 20 \\
Age, median years (range) & $31(18-60)$ \\
Male, $n$ (\%) & $6(30)$ \\
SCCAI, median (IQR) & $8(4)$ \\
CRP, median (IQR) & $4.0(12)$ \\
Hb, median (IQR) & $13.0(1.2)$ \\
Ferritin, median (IQR) & $43(58)$ \\
Platelets, median (IQR) & $328(193)$ \\
Baseline fecal calprotectin, median $\mu$ g/g (IQR) & $1801(1453)$ \\
Montreal classification, $n$ (\%) & \\
Left-sided colitis & $10(50)$ \\
$\quad$ Extensive colitis & $10(50)$ \\
Mayo endoscopic subscore, $n$ (\%) & \\
1 & $2(10)$ \\
2 & $9(45)$ \\
3 & $9(45)$ \\
Medical treatment, $n$ (\%) & \\
5-ASA & $20(100)$ \\
Prednisolone & $1(5)$ \\
Thiopurine & $4(20)$ \\
Methotrexate & $1(5)$ \\
Anti-TNF & $3(15)$ \\
\hline SCCAI: Simple Clinical Colitis Activity Index; IQR: interquartile range; CRP: &
\end{tabular}

Four patients had persistently elevated f-calprotectin and underwent a flexible sigmoidoscopy one year after the baseline colonoscopy. The results from follow-up of these patients are presented in Table 2.

Table 3 presents the sensitivity and specificity of two consecutive f-calprotectin $<250 \mu \mathrm{g} / \mathrm{g}$ as a surrogate marker of endoscopic mucosal healing.

\section{Discussion}

Two consecutive f-calprotectin $<250 \mu \mathrm{g} / \mathrm{g}$ predict endoscopic mucosal healing (Mayo endoscopic subscore of $\leq 1$ ) with a high positive predictive value, as demonstrated in this study. The negative predictive value was low, with wide confidence intervals, due to a limited sample size. Only one out of four patients with persisting high $\mathrm{f}$-calprotectin had not achieved endoscopic mucosal healing.

UC patients often report coinciding irritable bowel syndrome-like symptoms that might be misinterpreted as relapse or persisting disease activity [13-15]. SCCAI $<2.5$ are demonstrated to correlate with patient-defined remission with a sensitivity of $79 \%$ and a specificity of $82 \%$ [16]. In our study of patients achieving f-calprotectin $<250 \mu \mathrm{g} / \mathrm{g}$ and with endoscopic mucosal healing, there were still two patients with SCCAI $>2.5$. This is a challenge in clinical practice. In the European guidelines for endoscopy in inflammatory bowel disease (IBD), f-calprotectin is proposed as a potential surrogate marker of mucosal healing [17]. Our study supports this strategy, as f-calprotectin $<250 \mu \mathrm{g} / \mathrm{g}$ predicts endoscopic mucosal healing with high positive predictive value and may therefore be regarded as a reliable noninvasive biomarker.

As described previously, four out of 20 patients did not achieve two consecutive f-calprotectin values $<250 \mu \mathrm{g} / \mathrm{g}$ after standard medical treatment. All four patients were in clinical remission according to SCCAI. However, one patient still had endoscopic ulcerations corresponding to Mayo endoscopic subscore of 2. Such patients represent another challenge in clinical practice. The goal of treatment should be endoscopic mucosal healing; however, endoscopic monitoring is costly and unpopular amongst patients.

Elevated f-calprotectin is found to precede clinical relapse in clinically quiescent UC $[18,19]$. In a study of UC patients in endoscopic remission defined as Mayo endoscopic subscore of 0 , low $\mathrm{f}$-calprotectin predicted sustained remission better than absence of histological inflammation in colonic biopsies [20]. F-calprotectin is therefore suggested as a potential target of treatment itself.

A randomised case-control study has demonstrated that elevated f-calprotectin levels can be decreased by intensifying 5-ASA treatment in UC patients [21]. Another study evaluated 5-ASA dose escalation in UC patients monitored with monthly f-calprotectin measurements. UC patients in remission were randomised to a control group or an intervention group. The intervention group received dose escalation based on elevated f-calprotectin only. The authors found a tendency towards decrease of relapses in the intervention group [22]. Patients with active UC receiving medical treatment may also benefit from a similar strategy. f-calprotectin could be monitored and treatment evaluated and potentially intensified according to response.

Two consecutive elevated samples seem to be more accurate than one in predicting a forthcoming flare [23]. Intraindividual day-to-day $\mathrm{f}$-calprotectin variability has been described in patients without colonic inflammation and neoplasms ("patient controls") and in IBD patients [24-26]. This variation is even described within the same day in patients with active UC $[26,27]$, which may be confusing in clinical practice. However, we have previously demonstrated that f-calprotectin levels crossed proposed cut-off levels in only a minority of UC patients [28]. Nevertheless, one single f-calprotectin indicative of endoscopic mucosal healing should be confirmed with a consecutive control.

In the presented study, three out of four patients with persisting f-calprotectin $\geq 250 \mu \mathrm{g} / \mathrm{g}$ had Mayo endoscopic subscore of $\leq 1$ on the second endoscopy. These may be patients in risk of forthcoming flare. However, it is important to bear in mind that the endoscopic evaluation after treatment was a flexible sigmoidoscopy. F-calprotectin is a marker of inflammation throughout the entire gastrointestinal tract, and these patients may have had patchy inflammation due to a partial treatment response. A full colonoscopy could have clarified this.

A limitation in our study is the small sample size, hence wide confidence intervals for sensitivity and specificity. Our 


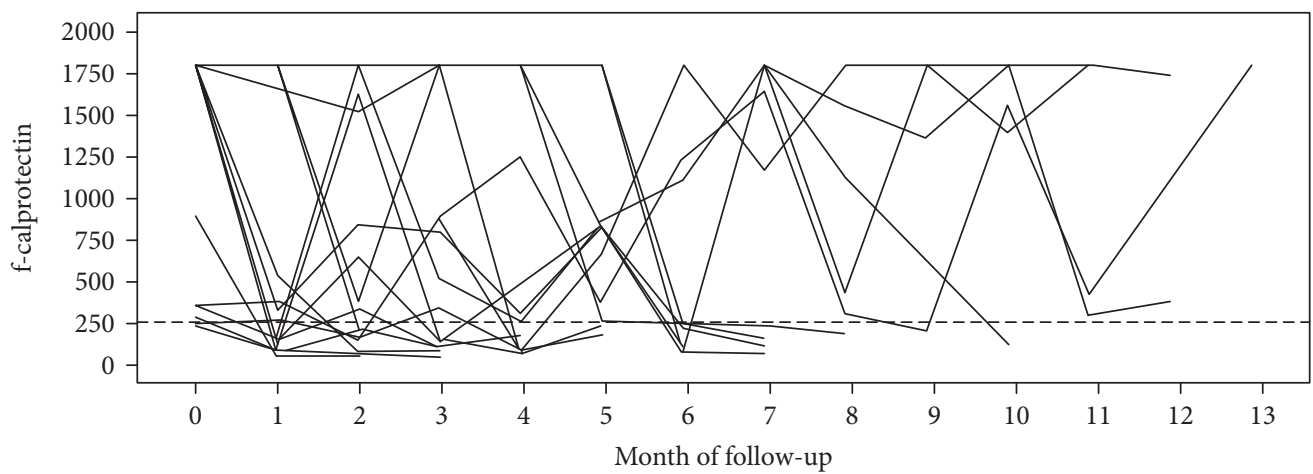

FIgure 2: The figure demonstrates how the f-calprotectin levels evolved within each study participant during the study period. Broken line represents the cut-off of $250 \mu \mathrm{g} / \mathrm{g}$. Within three months, six patients had values below cut-off. Within six months, 13 patients had values below cut-off. Within 10 months, 16 patients had values below cut-off. The remaining four patients never achieved values below cut-off.

TABLE 2: Results from follow-up of the four patients with persisting fecal calprotectin $>250 \mu \mathrm{g} / \mathrm{g}$ throughout the study period.

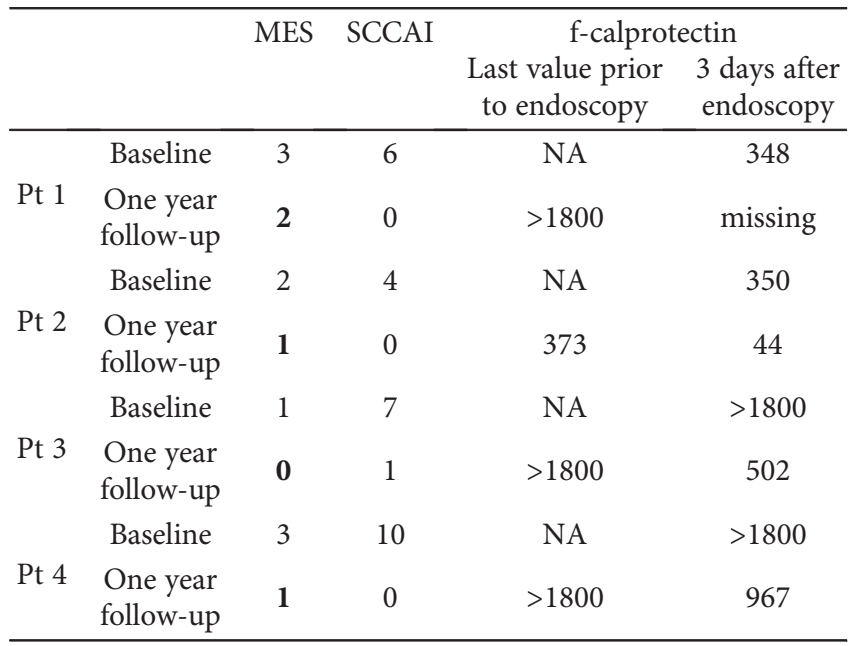

Pt: patient; MES: Mayo endoscopic subscore; SCCAI: Simple Clinical Colitis Activity Index.

TABle 3: Clinical performance characteristics of two consecutive fecal-calprotectin $<250 \mu \mathrm{g} / \mathrm{g}$ as a surrogate marker of mucosal healing after treatment for active ulcerative colitis.

\begin{tabular}{lc}
\hline Sensitivity (95\% CI) (\%) & $84.2(60.4-96.6)$ \\
Specificity (95\% CI) (\%) & $100(2.5-100)$ \\
PPV (95\% CI) (\%) & $100(79.4-100)$ \\
NPV (95\% CI) (\%) & $25.0(0.6-80.6)$ \\
\hline
\end{tabular}

CI: confidence interval; PPV: positive predictive value; NPV: negative predictive value.

study was single-center and exploratory, and a future larger multicenter study will therefore be appreciated.

In conclusion, f-calprotectin seems a useful marker of endoscopic mucosal healing after treatment for active UC. Further studies are needed to evaluate the clinical importance of high $\mathrm{f}$-calprotectin in patients in endoscopic remission.

\section{Conflicts of Interest}

Arne Røseth has received consultant fees from Bühlmann Laboratories AG, Switzerland. The other authors report no conflict of interest.

\section{Acknowledgments}

The authors would like to thank biostatistician Milada Cvancarova for the aid with the statistical analyses. This work was supported by research grants from Lovisenberg Diaconal Hospital.

\section{References}

[1] G. Meucci, R. Fasoli, S. Saibeni et al., "Prognostic significance of endoscopic remission in patients with active ulcerative colitis treated with oral and topical mesalazine: a prospective, multicenter study," Inflammatory Bowel Diseases, vol. 18, no. 6, pp. 1006-1010, 2012.

[2] S. Ardizzone, A. Cassinotti, P. Duca et al., "Mucosal healing predicts late outcomes after the first course of corticosteroids for newly diagnosed ulcerative colitis," Clinical Gastroenterology and Hepatology, vol. 9, no. 6, pp. 483-489.e3, 2011.

[3] K. F. Froslie, J. Jahnsen, B. A. Moum, M. H. Vatn, and IBSEN Group, "Mucosal healing in inflammatory bowel disease: results from a Norwegian population-based cohort," Gastroenterology, vol. 133, no. 2, pp. 412-422, 2007.

[4] T. Lobaton, F. Rodriguez-Moranta, A. Lopez, E. Sanchez, L. Rodriguez-Alonso, and J. Guardiola, "A new rapid quantitative test for fecal calprotectin predicts endoscopic activity in ulcerative colitis," Inflammatory Bowel Diseases, vol. 19, no. 5, pp. 1034-1042, 2013.

[5] A. M. Schoepfer, C. Beglinger, A. Straumann, M. Trummler, P. Renzulli, and F. Seibold, "Ulcerative colitis: correlation of the Rachmilewitz endoscopic activity index with fecal calprotectin, clinical activity, C-reactive protein, and blood leukocytes," Inflammatory Bowel Diseases, vol. 15, no. 12, pp. 1851-1858, 2009.

[6] A. M. Schoepfer, C. Beglinger, A. Straumann et al., "Fecal calprotectin more accurately reflects endoscopic activity of ulcerative colitis than the Lichtiger index, C-reactive protein, 
platelets, hemoglobin, and blood leukocytes," Inflammatory Bowel Diseases, vol. 19, no. 2, pp. 332-341, 2013.

[7] M. De Vos, O. Dewit, G. D'Haens et al., "Fast and sharp decrease in calprotectin predicts remission by infliximab in anti-TNF naive patients with ulcerative colitis," Journal of Crohn's \& Colitis, vol. 6, no. 5, pp. 557-562, 2012.

[8] D. Labaere, A. Smismans, A. Van Olmen et al., "Comparison of six different calprotectin assays for the assessment of inflammatory bowel disease," United European Gastroenterology Journal, vol. 2, no. 1, pp. 30-37, 2014.

[9] V. Kristensen, P. Klepp, M. Cvancarova, A. Roseth, V. Skar, and B. Moum, "Prediction of endoscopic disease activity in ulcerative colitis by two different assays for fecal calprotectin," Journal of Crohn's and Colitis, vol. 9, no. 2, pp. 164-169, 2015.

[10] J. Satsangi, M. S. Silverberg, S. Vermeire, and J. F. Colombel, "The Montreal classification of inflammatory bowel disease: controversies, consensus, and implications," Gut, vol. 55, no. 6, pp. 749-753, 2006.

[11] K. W. Schroeder, W. J. Tremaine, and D. M. Ilstrup, "Coated oral 5-aminosalicylic acid therapy for mildly to moderately active ulcerative colitis," New England Journal of Medicine, vol. 317, no. 26, pp. 1625-1629, 1987.

[12] R. S. Walmsley, R. C. Ayres, R. E. Pounder, and R. N. Allan, “A simple clinical colitis activity index," Gut, vol. 43, no. 1, pp. 2932, 1998.

[13] M. Simren, J. Axelsson, R. Gillberg, H. Abrahamsson, J. Svedlund, and E. S. Bjornsson, "Quality of life in inflammatory bowel disease in remission: the impact of IBS-like symptoms and associated psychological factors," The American Journal of Gastroenterology, vol. 97, no. 2, pp. 389-396, 2002.

[14] L. P. Jelsness-Jorgensen, T. Bernklev, and B. Moum, "Fatigue and disease-related worries among inflammatory bowel disease patients in remission; is it a reflection of coexisting IBSlike symptoms? A short report," Journal of Psychosomatic Research, vol. 73, no. 6, pp. 469-472, 2012.

[15] B. Jonefjall, H. Strid, L. Ohman, J. Svedlund, A. Bergstedt, and M. Simren, "Characterization of IBS-like symptoms in patients with ulcerative colitis in clinical remission," Neurogastroenterology \& Motility, vol. 25, no. 9, pp. 756-e578, 2013.

[16] P. D. Higgins, M. Schwartz, J. Mapili, I. Krokos, J. Leung, and E. M. Zimmermann, "Patient defined dichotomous end points for remission and clinical improvement in ulcerative colitis," Gut, vol. 54, no. 6, pp. 782-788, 2005.

[17] V. Annese, M. Daperno, M. D. Rutter et al., "European evidence based consensus for endoscopy in inflammatory bowel disease," Journal of Crohn's and Colitis, vol. 7, no. 12, pp. 982-1018, 2013.

[18] J. A. Tibble, G. Sigthorsson, S. Bridger, M. K. Fagerhol, and I. Bjarnason, "Surrogate markers of intestinal inflammation are predictive of relapse in patients with inflammatory bowel disease," Gastroenterology, vol. 119, no. 1, pp. 15-22, 2000.

[19] P. Molander, M. Farkkila, A. Ristimaki et al., "Does fecal calprotectin predict short-term relapse after stopping TNF $\alpha$ blocking agents in inflammatory bowel disease patients in deep remission?," Journal of Crohn's \& Colitis, vol. 9, no. 1, pp. 33-40, 2015.

[20] E. Mooiweer, M. Severs, M. E. Schipper et al., "Low fecal calprotectin predicts sustained clinical remission in inflammatory bowel disease patients: a plea for deep remission," Journal of Crohn's and Colitis, vol. 9, no. 1, pp. 50-55, 2015.
[21] M. T. Osterman, F. N. Aberra, R. Cross et al., "Mesalamine dose escalation reduces fecal calprotectin in patients with quiescent ulcerative colitis," Clinical Gastroenterology and Hepatology, vol. 12, no. 11, pp. 1887-1893.e3, 2014.

[22] A. Lasson, L. Ohman, P. O. Stotzer et al., "Pharmacological intervention based on fecal calprotectin levels in patients with ulcerative colitis at high risk of a relapse: a prospective, randomized, controlled study," United European Gastroenterology Journal, vol. 3, no. 1, pp. 72-79, 2015.

[23] M. De Vos, E. J. Louis, J. Jahnsen et al., "Consecutive fecal calprotectin measurements to predict relapse in patients with ulcerative colitis receiving infliximab maintenance therapy," Inflammatory Bowel Diseases, vol. 19, no. 10, pp. 2111-2117, 2013.

[24] B. Moum, J. Jahnsen, and T. Bernklev, "Fecal calprotectin variability in Crohn's disease," Inflammatory Bowel Diseases, vol. 16, no. 7, pp. 1091-1092, 2010.

[25] G. D. Naismith, L. A. Smith, S. J. Barry et al., “A prospective single-centre evaluation of the intra-individual variability of faecal calprotectin in quiescent Crohn's disease," Alimentary Pharmacology \& Therapeutics, vol. 37, no. 6, pp. 613-621, 2013.

[26] A. Lasson, P. O. Stotzer, L. Ohman, S. Isaksson, M. Sapnara, and H. Strid, "The intra-individual variability of faecal calprotectin: a prospective study in patients with active ulcerative colitis," Journal of Crohn's and Colitis, vol. 9, no. 1, pp. 2632, 2015.

[27] M. Calafat, E. Cabre, M. Manosa, T. Lobaton, L. Marin, and E. Domenech, "High within-day variability of fecal calprotectin levels in patients with active ulcerative colitis: what is the best timing for stool sampling?," Inflammatory Bowel Diseases, vol. 21, no. 5, pp. 1072-1076, 2015.

[28] V. Kristensen, G. H. Malmstrom, V. Skar, A. Roseth, and B. Moum, "Clinical importance of faecal calprotectin variability in inflammatory bowel disease: intra-individual variability and standardisation of sampling procedure," Scandinavian Journal of Gastroenterology, vol. 51, pp. 548-555, 2015. 


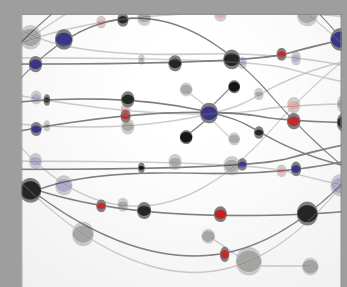

The Scientific World Journal
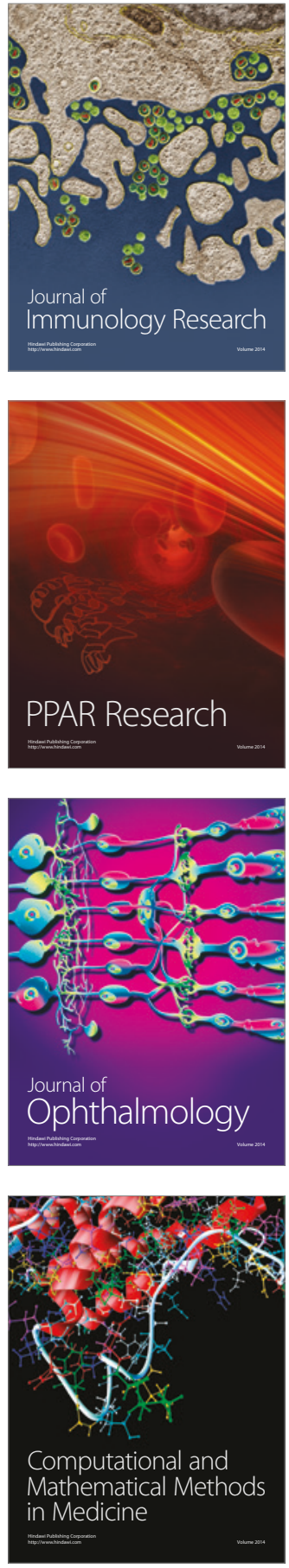

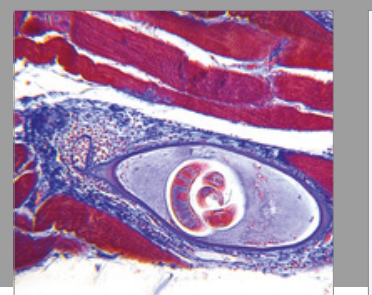

Gastroenterology Research and Practice
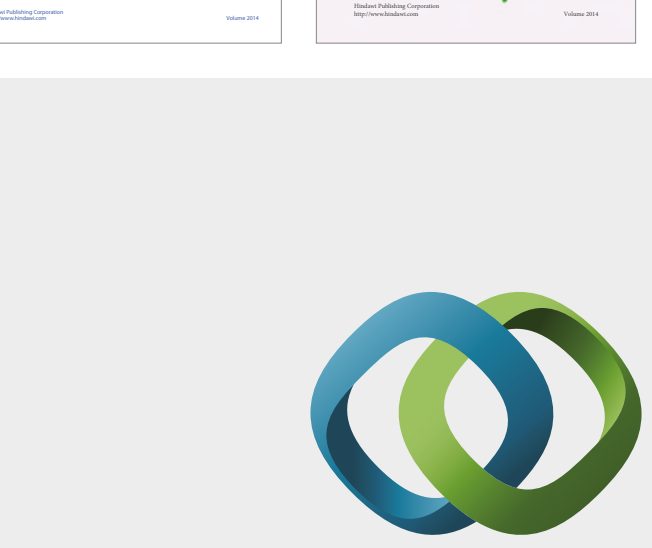

\section{Hindawi}

Submit your manuscripts at

https://www.hindawi.com
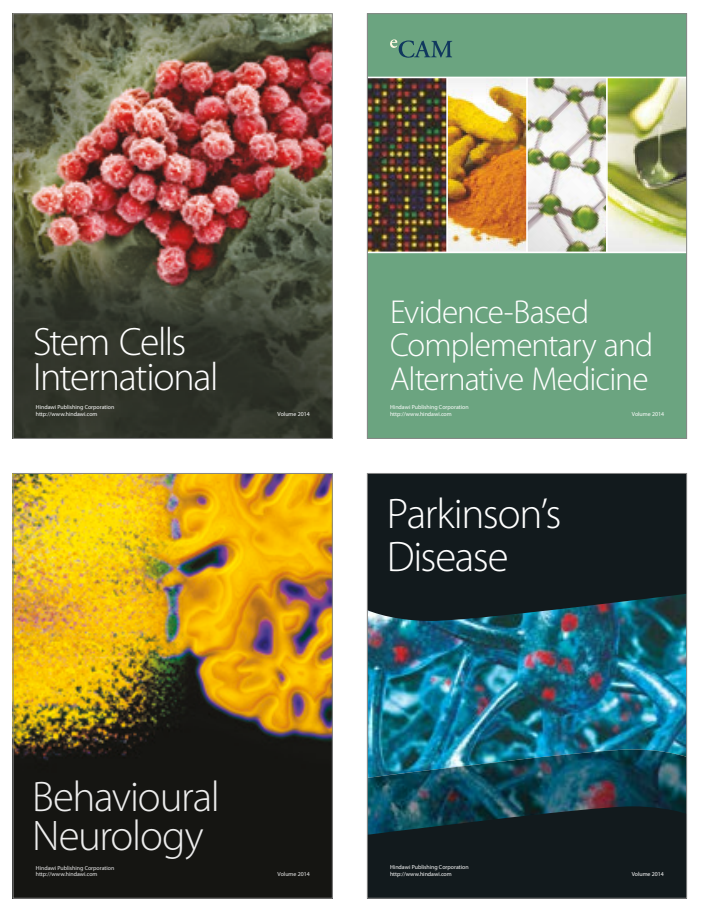
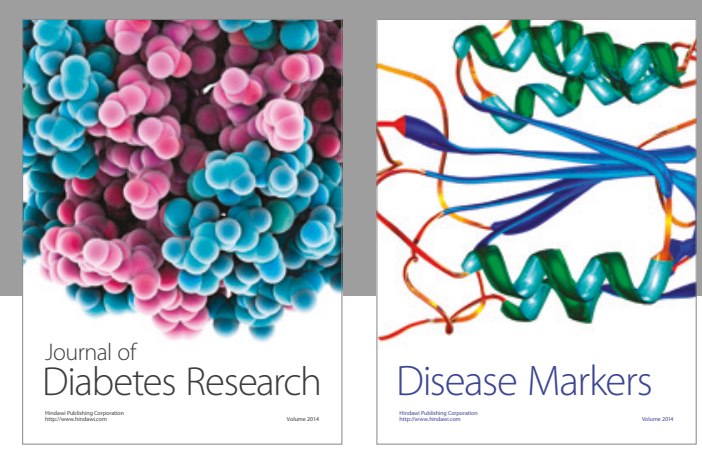

Disease Markers
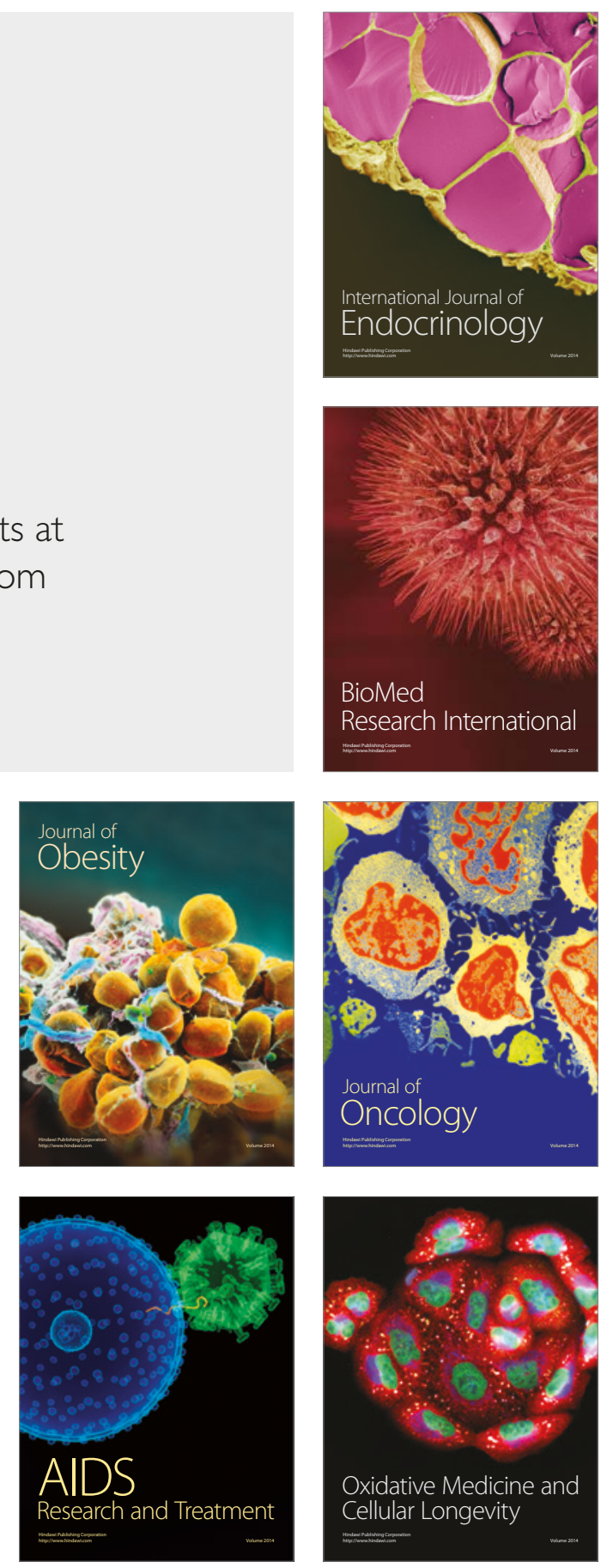